FACTA UNIVERSITATIS (NIŠ)

Ser. Math. Inform. Vol. 34, No 1 (2019), 149-164

https://doi.org/10.22190/FUMI1901149T

\title{
HERMITE-HADAMARD TYPE INEQUALITIES FOR P-CONVEX FUNCTIONS VIA KATUGAMPOLA FRACTIONAL INTEGRALS
}

\author{
Tekin Toplu, Erhan Set, İmdat İşcan and Selahattin Maden
}

\begin{abstract}
In this paper, the authors establish the Hermite-Hadamard inequality for p-convex functions via Katugampola fractional integrals, followed by proving a new identity involving Katugampola fractional integrals. By using this identity, some new Hermite-Hadamard type inequalities for classes of p-convex functions are obtained.

Keywords: p-convex function, Hermite-Hadamard type inequalities, Katugampola fractional integrals.
\end{abstract}

\section{Introduction}

Definition 1.1. The function $f: I \subset \mathbb{R} \rightarrow \mathbb{R}$, is said to be convex, if the following inequality holds

$$
f(t x+(1-t) y) \leq t f(x)+(1-t) f(y)
$$

for all $x, y \in I$ and $t \in[0,1]$. We say $f$ is concave if $(-f)$ is convex.

Now we will give a useful inequality for convex functions as below.

Let $f: I \subset \rightarrow \mathbb{R}$ be a convex function defined on an interval $I$ of real numbers and $a, b \in I$ with $a<b$. The following inequality

$$
f\left(\frac{a+b}{2}\right) \leq \frac{1}{b-a} \int_{a}^{b} f(x) d x \leq \frac{f(a)+f(b)}{2}
$$

holds. This double inequality is known in the literature as Hermite-Hadamard integral inequality for convex functions. Both inequalities hold in the reserved direction, when $f$ is concave. Hermite-Hadamard inequality for convex functions has

Received January, 17, 2018; Accepted January 18, 2019

2010 Mathematics Subject Classification. Primary 26D15; Secondary 26D10, 26A33 
received renewed attention in recent years and a remarkable variety of refinements and generalizations have been found; for example, see $[1,6,7,8,10,18,11]$ and the references cited therein.

In [28], Zhang and Wan gave a definition of the p-convex function as follows.

Definition 1.2. Let $I$ be a p-convex set. A function $f: I \rightarrow \mathbb{R}$ is said to be a p-convex function or belongs to class $P C(I)$, if

$$
f\left(\left[t x^{p}+(1-t) y^{p}\right]^{\frac{1}{p}}\right) \leq t f(x)+(1-t) f(y)
$$

for all $x, y \in I$ and $t \in[0,1]$.

Remark 1.1. [28] An interval $I$ is said to be a $p-$ convex set, if $\left[t x^{p}+(1-t) y^{p}\right]^{\frac{1}{p}} \in I$ for all $x, y \in I$ and $t \in[0,1]$, where $p=2 k+1$ or $p=n / m, n=2 r+1, m=2 s+1$ and $k, r, s \in \mathbb{N}$.

Remark 1.2. [9] If $I \subset(0, \infty)$ be a real interval and $p \in \mathbb{R} \backslash\{0\}$, then for all $x, y \in I$ and $t \in[0,1],\left[t x^{p}+(1-t) y^{p}\right]^{\frac{1}{p}} \in I$.

According to Remark 1.2, we can give a different version of the definition of the p-convex function as below.

Definition 1.3. [9] Let $I \subset(0, \infty)$ be a real interval and $p \in \mathbb{R} \backslash\{0\}$. A function $f: I \rightarrow \mathbb{R}$ is said to be a p-convex function, if

$$
f\left(\left[t x^{p}+(1-t) y^{p}\right]^{\frac{1}{p}}\right) \leq t f(x)+(1-t) f(y)
$$

for all $x, y \in I$ and $t \in[0,1]$. If the inequality is reserved, then $\mathrm{f}$ is said to be p-concave.

According to the definition above, it can easily be seen that for $p=1$ and $p=-1, \mathrm{p}$-convexity reduces to ordinary convexity and harmonically convexity [12] of functions defined on $I \subset(0, \infty)$ respectively.

In [3, Theorem 5], if we take $I \subset(0, \infty), p \in \mathbb{R} \backslash\{0\}$ and $h(t)=t$, then we have the following inequalities for $p$-convex functions. have

$f: I \rightarrow \mathbb{R}$ be a p-convex function and $a, b \in I$ with $a<b$. If $f \in L[a, b]$, then we

$$
f\left(\left[\frac{a^{p}+b^{p}}{2}\right]^{\frac{1}{p}}\right) \leq \frac{p}{b^{p}-a^{p}} \int_{a}^{b} \frac{f(x)}{x^{1-p}} d x \leq \frac{f(a)+f(b)}{2} .
$$

For some results related to p-convex functions and its generalizations, we refer the reader to see now $[3,9,22,21,28]$.

In [22, Lemma 2.4], if we take $I \subset(0, \infty)$ and $p \in \mathbb{R} \backslash\{0\}$, then we have the following Lemma. 
Lemma 1.1. Let $f: I \rightarrow \mathbb{R}$ be a differentiable function on $I^{\circ}$ and $a, b \in I$ with $a<b$. If $f^{\prime} \in L[a, b]$, then we have,

$$
\begin{aligned}
& \frac{f(a)+f(b)}{2}-\frac{p}{b^{p}-a^{p}} \int_{b}^{a} \frac{f(x)}{x^{1-p}} d x \\
= & \frac{p}{b^{p}-a^{p}} \int_{0}^{1} \frac{1-2 t}{\left[t a^{p}+(1-t) b^{p}\right]^{1-\frac{1}{p}}} f^{\prime}\left(\left[t a^{p}+(1-t) b^{p}\right]^{\frac{1}{p}}\right) d t .
\end{aligned}
$$

We recall the following special functions and inequality.(see [16, 27] )

(1) The Gamma Function:

The Gamma $\Gamma$ function is defined by

$$
\Gamma(z)=\Gamma(\alpha)=\int_{0}^{\infty} e^{-t} t^{\alpha-1} d t
$$

for all complex numbers $z$ with $\operatorname{Re}(z)>0$, respectively. The gamma function is a natural extension of the factorial from integers $n$ to real (and complex) numbers $z$.

(2) The Beta Function:

$$
\beta(x, y)=\frac{\Gamma(x) \Gamma(y)}{\Gamma(x+y)}=\int_{0}^{1} t^{x-1}(1-t)^{y-1} d t, \quad x, y>0
$$

(3) The Hypergeometric Function

$$
{ }_{2} F_{1}(a, b ; c, z)=\frac{1}{\beta(b, c-b)} \int_{0}^{1} t^{b-1}(1-t)^{c-b-1}(1-z t)^{-a} d t, c>b>0,|z|<1 .
$$

Lemma 1.2. [24, 29] For $0<\alpha<1$ and $0 \leq a<b$, we have

$$
\left|a^{\alpha}-b^{\alpha}\right| \leq(b-a)^{\alpha} \text {. }
$$

Definition 1.4. Let $[a, b]$ be a finite interval on the real axis $\mathbb{R}$ and $f \in L[a, b]$. The Riemann-Liouville fractional integrals $J_{a^{+}}^{\alpha} f$ and $J_{b^{-}}^{\alpha} f$ of order $\alpha>0$ are defined by

$$
\begin{aligned}
J_{a^{+}}^{\alpha} f(x) & =\frac{1}{\Gamma(\alpha)} \int_{a}^{x}(x-t)^{\alpha-1} f(t) d t, \quad x>a, \\
J_{b^{-}}^{\alpha} f(x) & =\frac{1}{\Gamma(\alpha)} \int_{x}^{b}(t-x)^{\alpha-1} f(t) d t, \quad x<b
\end{aligned}
$$

respectively.(see [16]) 
In [26] Sarıkaya et al. proved the following theorem for Riemann-Liouville fractional integrals.

Theorem 1.1. Let $f:[a, b] \rightarrow \mathbb{R}$ be a positive function with $0 \leq a<b$ and $f \in$ $L_{1}[a, b]$. If $f$ is convex function on $[a, b]$, then the following inequality for fractional integrals holds:

$$
f\left(\frac{a+b}{2}\right) \leq \frac{\Gamma(\alpha+1)}{2(b-a)^{\alpha}}\left[J_{a^{+}}^{\alpha} f(b)+J_{b^{-}}^{\alpha} f(a)\right] \leq \frac{f(a)+f(b)}{2}
$$

with $\alpha>0$

Definition 1.5. [17] Let the space $X_{c}^{p}(a, b)(c \in \mathbb{R}, 1 \leq p \leq \infty)$ of those complexvalued Lebesque measurable functions $f$ on $[a, b]$ for which $\|f\| x_{c}^{p}<\infty$, where the norm is defined by,

$$
\|f\| x_{c}^{p}-\left(\int_{a}^{b}\left|t^{c} f(t)\right|^{p} \frac{d t}{t}\right)^{1 / p}<\infty
$$

for $1 \leq p \leq \infty, c \in \mathbb{R}$ and for the case $p=\infty$,

$$
\|f\| x_{c}^{p}=e s s \sup _{a \leq t \leq b}\left[t^{c}|f(t)|\right] \quad(c \in \mathbb{R}) .
$$

Katugampola introduced a new fractional which generalizes the Riemann-Liouville and the Hadamard fractional integrals into a single form as follows.(see $[13,14,15]$ )

Definition 1.6. Let $[a, b] \subset \mathbb{R}$ be a finite interval. Then, the left-and right-side Katugampola fractional integrals of order $(\alpha>0)$ of $f \in X_{c}^{p}(a, b)$ are defined by

${ }^{p} I_{a^{+}}^{\alpha} f(x)=\frac{p^{1-\alpha}}{\Gamma(\alpha)} \int_{a}^{x} \frac{t^{p-1}}{\left(x^{p}-t^{p}\right)^{1-\alpha}} f(t) d t \quad$ and ${ }^{p} I_{b^{-}}^{\alpha} f(x)=\frac{p^{1-\alpha}}{\Gamma(\alpha)} \int_{x}^{b} \frac{t^{p-1}}{\left(t^{p}-x^{p}\right)^{1-\alpha}} f(t) d t$

with $a<x<b$ and $p>0$, if the integral exists.

For more detailed information about fractional integrals and their applications, we refer the reader to see $[4,5,2,20,23,25,19]$

The aim of this paper is to establish some new Hermite-Hadamard type inequalities for $p$-convex function via Katugampola fractional integral.

\section{Main Results}

Let $f: I \subset(0, \infty) \rightarrow \mathbb{R}$ be a differentiable function on $I^{\circ}$, the interior of $I$, throughout this section,

$$
K_{f}(\alpha, a, b)=\frac{f(a)+f(b)}{2}-\frac{p^{\alpha} \Gamma(\alpha+1)}{2\left(b^{p}-a^{p}\right)^{\alpha}}\left[{ }^{p} I_{a^{+}}^{\alpha} f(b)+{ }^{p} I_{b^{-}}^{\alpha} f(a)\right]
$$

will be taken, where $a, b \in I, \alpha>0$ and $\Gamma$ is Euler Gamma function. 
Theorem 2.1. Let $f: I \subset(0, \infty) \rightarrow \mathbb{R}$ be a p-convex function, $p>0, \alpha>0$ and $a, b \in I$ with $a<b$. If $f \in L[a, b]$, then the following inequality for fractional integrals holds:

$$
f\left(\left[\frac{a^{p}+b^{p}}{2}\right]^{\frac{1}{p}}\right) \leq \frac{p^{\alpha} \Gamma(\alpha+1)}{2\left(b^{p}-a^{p}\right)^{\alpha}}\left[{ }^{p} I_{a^{+}}^{\alpha} f(b)+{ }^{p} I_{b^{-}}^{\alpha} f(a)\right] \leq \frac{f(a)+f(b)}{2}
$$

Proof. Since $f$ is p-convex function on $[a, b]$, we have for all $x, y \in[a, b]$ (with $t=\frac{1}{2}$ in 1.2 )

$$
f\left(\left[\frac{x^{p}+y^{p}}{2}\right]^{\frac{1}{p}}\right) \leq \frac{f(x)+f(y)}{2} .
$$

By choosing $x=\left[t a^{p}+(1-t) b^{p}\right]^{\frac{1}{p}}$ and $y=\left[(1-t) a^{p}+t b^{p}\right]^{\frac{1}{p}}$, then we get

$$
2 f\left(\left[\frac{a^{p}+b^{p}}{2}\right]^{\frac{1}{p}}\right) \leq f\left(\left[t a^{p}+(1-t) b^{p}\right]^{\frac{1}{p}}\right)+f\left(\left[(1-t) a^{p}+t b^{p}\right]^{\frac{1}{p}}\right) .
$$

Multiplying both sides of the inequality of (2.2) by $t^{\alpha-1}$ and then integrating the resulting inequality with respect to $t$ over $[0,1]$, then we obtain,

$$
\begin{aligned}
\frac{2}{\alpha} f\left(\left[\frac{a^{p}+b^{p}}{2}\right]^{\frac{1}{p}}\right) \leq & \int_{0}^{1} t^{\alpha-1} f\left(\left[t a^{p}+(1-t) b^{p}\right]^{\frac{1}{p}}\right) d t \\
& +\int_{0}^{1} t^{\alpha-1} f\left(\left[(1-t) a^{p}+t b^{p}\right]^{\frac{1}{p}}\right) d t \\
= & \int_{b}^{a}\left(\frac{b^{p}-x^{p}}{b^{p}-a^{p}}\right)^{\alpha-1} f(x) \frac{p x^{p-1}}{a^{p}-b^{p}} d x \\
& +\int_{a}^{b}\left(\frac{x^{p}-a^{p}}{b^{p}-a^{p}}\right)^{\alpha-1} f(x) \frac{p x^{p-1}}{b^{p}-a^{p}} d x \\
= & \frac{p^{\alpha} \Gamma(\alpha)}{\left(b^{p}-a^{p}\right)^{\alpha}}\left[{ }^{p} I^{\alpha+} f(b)+{ }^{p} I_{b^{-}}^{\alpha} f(a)\right] .
\end{aligned}
$$

Thus we have

$$
f\left(\left[\frac{a^{p}+b^{p}}{2}\right]^{\frac{1}{p}}\right) \leq \frac{p^{\alpha} \Gamma(\alpha+1)}{2\left(b^{p}-a^{p}\right)^{\alpha}}\left[{ }^{p} I_{a^{+}}^{\alpha} f(b)+{ }^{p} I_{b^{-}}^{\alpha} f(a)\right],
$$

which completes the proof of the the first inequality. For the proof of the second inequality in (2.1), by using p-convexity of a function $f$, we can write,

$$
f\left(\left[t a^{p}+(1-t) b^{p}\right]^{\frac{1}{p}}\right) \leq t f(a)+(1-t) f(b),
$$


and

$$
f\left(\left[(1-t) a^{p}+t b^{p}\right]^{\frac{1}{p}}\right) \leq(1-t) f(a)+t f(b) .
$$

By adding these inequalities, then we have,

$$
f\left(\left[t a^{p}+(1-t) b^{p}\right]^{\frac{1}{p}}\right)+f\left(\left[(1-t) a^{p}+t b^{p}\right]^{\frac{1}{p}}\right) \leq f(a)+f(b) .
$$

Multiplying both sides of the equation (2.3) by $t^{\alpha-1}, \alpha>0$ and then integrating the resulting inequality with $t$ over $[0,1]$, we similarly obtain,

$$
\frac{p^{\alpha} \Gamma(\alpha+1)}{2\left(b^{p}-a^{p}\right)^{\alpha}}\left[{ }^{p} I_{a^{+}}^{\alpha} f(b)+{ }^{p} I_{b^{-}}^{\alpha} f(a)\right] \leq \frac{f(a)+f(b)}{2} .
$$

So the proof is completed.

Remark 2.1. In Theorem 2.1, if we take $p=1$, then the inequality reduces to the inequality (1.5).

Remark 2.2. In Theorem 2.1, if we take $\alpha=1$, then the inequality reduces to the inequality (1.3).

Lemma 2.1. Let $f: I \subset(0, \infty) \rightarrow \mathbb{R}$ be a differentiable function mapping with $0 \leq a<b$. If $f^{\prime}$ is differentiable on $[a, b]$, then the following inequality holds:

$$
K_{f}(\alpha, a, b)=\frac{b^{p}-a^{p}}{2 p} \int_{0}^{1} \frac{\left[(1-t)^{\alpha}-t^{\alpha}\right] f^{\prime}\left(\left[t a^{p}+(1-t) b^{p}\right]^{\frac{1}{p}}\right)}{\left[t a^{p}+(1-t) b^{p}\right]^{1-\frac{1}{p}}} d t .
$$

Proof. Let $M_{p}=t a^{p}+(1-t) b^{p}$. It suffices to note that

$$
\begin{aligned}
& K_{f}(\alpha, a, b) \\
= & \frac{b^{p}-a^{p}}{2 p} \int_{0}^{1} \frac{\left[(1-t)^{\alpha}-t^{\alpha}\right] f^{\prime}\left(\left[t a^{p}+(1-t) b^{p}\right]^{\frac{1}{p}}\right)}{\left[t a^{p}+(1-t) b^{p}\right]^{1-\frac{1}{p}}} d t \\
= & \frac{b^{p}-a^{p}}{2 p} \int_{0}^{1} \frac{(1-t)^{\alpha} f^{\prime}\left(\left[t a^{p}+(1-t) b^{p}\right]^{\frac{1}{p}}\right)}{\left[t a^{p}+(1-t) b^{p}\right]^{1-\frac{1}{p}}} d t \\
= & -\frac{b^{p}-a^{p}}{2 p} \int_{0}^{1} \frac{t^{\alpha} f^{\prime}\left(\left[t a^{p}+(1-t) b^{p}\right]^{\frac{1}{p}}\right)}{\left[t a^{p}+(1-t) b^{p}\right]^{1-\frac{1}{p}}} d t \\
= & I_{1}+I_{2} .
\end{aligned}
$$

By integrating the part, we have,

$$
I_{1}=-\frac{1}{2}\left[\begin{array}{c}
\left.(1-t)^{\alpha-1} f\left(\left[t a^{p}+(1-t) b^{p}\right]^{\frac{1}{p}}\right)\right|_{0} ^{1} \\
+\alpha \int_{0}^{1}(1-t)^{\alpha-1} f\left(\left[t a^{p}+(1-t) b^{p}\right]^{\frac{1}{p}}\right) d t
\end{array}\right],
$$


if we take $x=\left[t a^{p}+(1-t) b^{p}\right]^{\frac{1}{p}}$

$$
\begin{aligned}
& =-\frac{1}{2}\left[-f(b)+\frac{p \alpha}{\left(b^{p}-a^{p}\right)^{\alpha}} \int_{a}^{b} \frac{\left(x^{p}-a^{p}\right)^{\alpha-1}}{x^{1-p}} f(x) d x\right] \\
& =\frac{f(b)}{2}-\frac{p \alpha}{2\left(b^{p}-a^{p}\right)^{\alpha}} \int_{a}^{b} \frac{\left(x^{p}-a^{p}\right)^{\alpha-1}}{x^{1-p}} f(x) d x \\
& =\frac{f(b)}{2}-\frac{p^{\alpha} \Gamma(\alpha+1)}{2\left(b^{p}-a^{p}\right)^{\alpha}}\left[{ }^{p} I_{b^{-}}^{\alpha} f(a)\right]
\end{aligned}
$$

and similarly we get $I_{2}$,

$$
\begin{aligned}
I_{2} & =\frac{1}{2}\left[\left.t^{\alpha-1} f\left(\left[t a^{p}+(1-t) b^{p}\right]^{\frac{1}{p}}\right)\right|_{0} ^{1}+\alpha \int_{0}^{1} t^{\alpha-1} f\left(\left[t a^{p}+(1-t) b^{p}\right]^{\frac{1}{p}}\right) d t\right] \\
& =\frac{1}{2}\left[-f(a)-\frac{p \alpha}{\left(b^{p}-a^{p}\right)^{\alpha}} \int_{a}^{b} \frac{\left(b^{p}-a^{p}\right)^{\alpha-1}}{x^{1-p}} f(x) d x\right] \\
& =\frac{f(a)}{2}-\frac{p \alpha}{2\left(b^{p}-a^{p}\right)^{\alpha}} \int_{a}^{b} \frac{\left(b^{p}-x^{p}\right)^{\alpha-1}}{x^{1-p}} f(x) d x \\
& =\frac{f(a)}{2}-\frac{p^{\alpha} \Gamma(\alpha+1)}{2\left(b^{p}-a^{p}\right)^{\alpha}}\left[{ }^{p} I_{a^{+}}^{\alpha} f(b)\right] .
\end{aligned}
$$

By adding the results of (2.6) and (2.7) side by side in the equation (2.6), we obtain the inequality (2.4). This completes the proof.

Remark 2.3. Also in the equation (2.4) of Lemma (2.1), if we take specially $\alpha=1$, then the inequality reduces to the equation (1.4).

By using Lemma 2.1, we can have the following fractional inequality.

Theorem 2.2. Let $f: I \subset(0, \infty) \rightarrow \mathbb{R}$ be a differentiable function on $I^{\circ}, a, b \in I$ with $a<b, p>0$, and $f^{\prime} \in L[a, b]$. If $\left|f^{\prime}\right|^{q}$ is $p$-convex on $[a, b]$ for $q \geq 1$ then the following inequality for fractional integrals holds:

$$
\left|K_{f}(\alpha, a, b)\right| \leq \frac{b^{p}-a^{p}}{2 p} M_{1}^{1-1 / q}(\alpha, a, b)\left[M_{2}(\alpha, a, b)\left|f^{\prime}(a)\right|^{q}+M_{3}(\alpha, a, b)\left|f^{\prime}(b)\right|^{q}\right]^{1 / q}
$$

where

$$
M_{1}(\alpha, a, b)=\frac{b^{1-p}}{\alpha+1}\left[\begin{array}{c}
{ }_{2} F_{1}\left(1-\frac{1}{p}, 1 ; \alpha+2 ; 1-\frac{a^{p}}{b^{p}}\right) \\
+{ }_{2} F_{1}\left(1-\frac{1}{p}, \alpha+1 ; \alpha+2 ; 1-\frac{a^{p}}{b^{p}}\right)
\end{array}\right]
$$




$$
\begin{aligned}
& M_{2}(\alpha, a, b)=\frac{b^{1-p}}{\alpha+2}\left[\begin{array}{c}
\frac{1}{\alpha+1}{ }_{2} F_{1}\left(1-\frac{1}{p}, 2 ; \alpha+3 ; 1-\frac{a^{p}}{b^{p}}\right) \\
+{ }_{2} F_{1}\left(1-\frac{1}{p}, \alpha+2 ; \alpha+3 ; 1-\frac{a^{p}}{b^{p}}\right)
\end{array}\right] \\
& M_{3}(\alpha, a, b)=\frac{b^{1-p}}{\alpha+1}\left[\begin{array}{c}
{ }_{2} F_{1}\left(1-\frac{1}{p}, 1 ; \alpha+3 ; 1-\frac{a^{p}}{b^{p}}\right) \\
+\frac{1}{\alpha+1}{ }_{2} F_{1}\left(1-\frac{1}{p}, \alpha+1 ; \alpha+3 ; 1-\frac{a^{p}}{b^{p}}\right)
\end{array}\right] .
\end{aligned}
$$

Proof. From Lemma 2.1 by using the property of the modulus, the power mean inequality and the p-convexity of $\left|f^{\prime}\right|^{q}$, then we have,

$$
\begin{aligned}
(2.9) \leq & \frac{K_{f}(\alpha, a, b) \mid}{2 p} \int_{0}^{1} \frac{\left|(1-t)^{\alpha}-t^{\alpha}\right| \mid f^{\prime}\left(\left[t a^{p}+(1-t) b^{p}\right]\right.}{\left[t a^{p}+(1-t) b^{p}\right]^{1-\frac{1}{p}}} d t \\
\leq & \frac{b^{p}-a^{p}}{2 p}\left(\int_{0}^{1} \frac{\left|(1-t)^{\alpha}-t^{\alpha}\right|}{\left[t a^{p}+(1-t) b^{p}\right]^{1-\frac{1}{p}}} d t\right)^{1-1 / q} \\
& \times\left(\int_{0}^{1} \frac{\left|(1-t)^{\alpha}-t^{\alpha}\right|\left|f^{\prime}\left(\left[t a^{p}+(1-t) b^{p}\right]^{\frac{1}{p}}\right)\right|^{q}}{\left[t a^{p}+(1-t) b^{p}\right]^{1-\frac{1}{p}}} d t\right)^{1 / q} \\
\leq & \left.\frac{b^{p}-a^{p}}{2 p}\left(\int_{0}^{1} \frac{\left[(1-t)^{\alpha}+t^{\alpha}\right]}{\left[t a^{p}+(1-t) b^{p}\right]^{1-\frac{1}{p}}} d t\right)^{1-1 / q}\right)^{1} \\
& \times\left(\int_{0}^{1} \frac{\left[(1-t)^{\alpha}+t^{\alpha}\right]}{\left.\left[t a^{p}+(1-t) b^{p}\right]^{1-\frac{1}{p}}\left[t\left|f^{\prime}(a)\right|^{q}+(1-t) t\left|f^{\prime}(b)\right|^{q}\right] d t\right)^{1 / q}}\right. \\
& \frac{b^{p}-a^{p}}{2 p} M_{1}^{1-1 / q}(\alpha, a, b)\left[M_{2}(\alpha, a, b)\left|f^{\prime}(a)\right|^{q}+M_{3}(\alpha, a, b)\left|f^{\prime}(b)\right|^{q}\right]^{1 / q}, \\
(2.10)= &
\end{aligned}
$$

where, by simple computation, we obtain,

$$
\begin{aligned}
M_{1}(\alpha, a, b) & =\int_{0}^{1} \frac{\left[(1-t)^{\alpha}+t^{\alpha}\right]}{\left[t a^{p}+(1-t) b^{p}\right]^{1-\frac{1}{p}}} d t \\
& =\frac{b^{1-p}}{\alpha+1}\left[\begin{array}{c}
{ }_{2} F_{1}\left(1-\frac{1}{p}, 1 ; \alpha+2 ; 1-\frac{a^{p}}{b^{p}}\right) \\
+{ }_{2} F_{1}\left(1-\frac{1}{p}, \alpha+1 ; \alpha+2 ; 1-\frac{a^{p}}{b^{p}}\right)
\end{array}\right] \\
M_{2}(\alpha, a, b) & =\int_{0}^{1} \frac{\left[(1-t)^{\alpha}+t^{\alpha}\right]}{\left[t a^{p}+(1-t) b^{p}\right]^{1-\frac{1}{p}}} t d t
\end{aligned}
$$




$$
=\frac{b^{1-p}}{\alpha+2}\left[\begin{array}{c}
\frac{1}{\alpha+1}{ }_{2} F_{1}\left(1-\frac{1}{p}, 2 ; \alpha+3 ; 1-\frac{a^{p}}{b^{p}}\right) \\
+{ }_{2} F_{1}\left(1-\frac{1}{p}, \alpha+2 ; \alpha+3 ; 1-\frac{a^{p}}{b^{p}}\right)
\end{array}\right]
$$

$$
\begin{aligned}
M_{3}(\alpha, a, b) & =\int_{0}^{1} \frac{\left[(1-t)^{\alpha}+t^{\alpha}\right]}{\left[t a^{p}+(1-t) b^{p}\right]^{1-\frac{1}{p}}}(1-t) d t \\
& =\frac{b^{1-p}}{\alpha+1}\left[\begin{array}{c}
{ }_{2} F_{1}\left(1-\frac{1}{p}, 1 ; \alpha+3 ; 1-\frac{a^{p}}{b^{p}}\right) \\
+\frac{1}{\alpha+1}_{2} F_{1}\left(1-\frac{1}{p}, \alpha+1 ; \alpha+3 ; 1-\frac{a^{p}}{b^{p}}\right)
\end{array}\right] .
\end{aligned}
$$

Then by using the results from the equations (2.11)-(2.13) in the equation (2.10), we have desired result (2.9). This completes the proof.

Remark 2.4. If we specially take $\alpha=1$, in inequality 2.9 , then the inequality reduces to $[22$, Theorem 3.2].

When $0<\alpha \leq 1$ by using Lemma 1.2 and Lemma 2.1, we have another fractional integral inequality for $\mathrm{p}$ convex functions as follows.

Theorem 2.3. Let $f: I \subset(0, \infty) \rightarrow \mathbb{R}$ be a differentiable function on $I^{\circ}, a, b \in I$ with $a<b, p>0$, and $f^{\prime} \in L[a, b]$. If $\left|f^{\prime}\right|^{q}$ is $p$-convex on $[a, b]$ for $q \geq 1$, then the following inequality for fractional integrals holds:

$$
\left|K_{f}(\alpha, a, b)\right| \leq \frac{b^{p}-a^{p}}{2 p} M_{4}^{1-1 / q}(\alpha, a, b)\left[M_{5}(\alpha, a, b)\left|f^{\prime}(a)\right|^{q}+M_{6}(\alpha, a, b)\left|f^{\prime}(b)\right|^{q}\right]^{1 / q}
$$

where

$$
\begin{aligned}
& M_{4}(\alpha, a, b)=\frac{b^{1-p}}{\alpha+1}\left[\begin{array}{c}
{ }_{2} F_{1}\left(1-\frac{1}{p}, \alpha+1 ; \alpha+2 ; 1-\frac{a^{p}}{b^{p}}\right) \\
-{ }_{2} F_{1}\left(1-\frac{1}{p}, 1 ; \alpha+2 ; 1-\frac{a^{p}}{b^{p}}\right) \\
+{ }_{2} F_{1}\left(1-\frac{1}{p}, 1 ; \alpha+2 ; \frac{1}{2}\left(1-\frac{a^{p}}{b^{p}}\right)\right)
\end{array}\right] \\
& M_{5}(\alpha, a, b)=\frac{b^{1-p}}{\alpha+2}\left[\begin{array}{c}
{ }_{2} F_{1}\left(1-\frac{1}{p}, \alpha+2 ; \alpha+3 ; 1-\frac{a^{p}}{b^{p}}\right) \\
-\frac{1}{\alpha+1} 2 F_{1}\left(1-\frac{1}{p}, 2 ; \alpha+3 ; 1-\frac{a^{p}}{b^{p}}\right) \\
+\frac{1}{2(\alpha+1)} F_{1}\left(1-\frac{1}{p}, 2 ; \alpha+3 ; \frac{1}{2}\left(1-\frac{a^{p}}{b^{p}}\right)\right)
\end{array}\right] \\
& M_{6}(\alpha, a, b)=\frac{b^{1-p}}{\alpha+2}\left[\begin{array}{c}
\frac{1}{\alpha+1} F_{1}\left(1-\frac{1}{p}, \alpha+1 ; \alpha+3 ; 1-\frac{a^{p}}{b^{p}}\right) \\
-{ }_{2} F_{1}\left(1-\frac{1}{p}, 1 ; \alpha+3 ; 1-\frac{a^{p}}{b^{p}}\right) \\
+{ }_{2} F_{1}\left(1-\frac{1}{p}, 1 ; \alpha+2 ; \frac{1}{2}\left(1-\frac{a^{p}}{b^{p}}\right)\right) \\
-\frac{1}{2(\alpha+1)} F_{2}\left(1-\frac{1}{p}, 2 ; \alpha+3 ; \frac{1}{2}\left(1-\frac{a^{p}}{b^{p}}\right)\right)
\end{array}\right]
\end{aligned}
$$

and $0<\alpha \leq 1$. 
Proof. From Lemma 2.1 using the property of the modulus, the power mean inequality and the p-convexity of $\left|f^{\prime}\right|^{q}$, we have,

$$
\begin{aligned}
& \left|K_{f}(\alpha, a, b)\right| \\
\leq & \frac{b^{p}-a^{p}}{2 p} \int_{0}^{1} \frac{\left|(1-t)^{\alpha}-t^{\alpha}\right|\left|f^{\prime}\left(\left[t a^{p}+(1-t) b^{p}\right]^{\frac{1}{p}}\right)\right|}{\left[t a^{p}+(1-t) b^{p}\right]^{1-\frac{1}{p}}} d t \\
\leq & \frac{b^{p}-a^{p}}{2 p}\left(\int_{0}^{1} \frac{\left|(1-t)^{\alpha}-t^{\alpha}\right|}{\left[t a^{p}+(1-t) b^{p}\right]^{1-\frac{1}{p}}} d t\right)^{1-1 / q} \\
& \times\left(\int_{0}^{1} \frac{\left|(1-t)^{\alpha}-t^{\alpha}\right|\left|f^{\prime}\left(\left[t a^{p}+(1-t) b^{p}\right]^{\frac{1}{p}}\right)\right|^{q}}{\left[t a^{p}+(1-t) b^{p}\right]^{1-\frac{1}{p}}} d t\right)^{1 / q}
\end{aligned}
$$

$$
\begin{aligned}
\leq & \frac{b^{p}-a^{p}}{2 p}\left(\int_{0}^{1} \frac{\left|(1-t)^{\alpha}-t^{\alpha}\right|}{\left[t a^{p}+(1-t) b^{p}\right]^{1-\frac{1}{p}}} d t\right)^{1-1 / q} \\
& \times\left(\int_{0}^{1} \frac{\left|(1-t)^{\alpha}-t^{\alpha}\right|}{\left[t a^{p}+(1-t) b^{p}\right]^{1-\frac{1}{p}}}\left[t\left|f^{\prime}(a)\right|^{q}+(1-t) t\left|f^{\prime}(b)\right|^{q}\right] d t\right)^{1 / q} \\
(2.17)= & \frac{b^{p}-a^{p}}{2 p} K_{4}^{1-1 / q}(\alpha, a, b)\left[K_{5}(\alpha, a, b)\left|f^{\prime}(a)\right|^{q}+K_{6}(\alpha, a, b)\left|f^{\prime}(b)\right|^{q}\right]^{1 / q},
\end{aligned}
$$

where by using Lemma 1.2 and by simple calculations of integrals, we obtain,

$$
\begin{aligned}
K_{4}= & \int_{0}^{1} \frac{\left|(1-t)^{\alpha}-t^{\alpha}\right|}{\left[t a^{p}+(1-t) b^{p}\right]^{1-\frac{1}{p}}} d t \\
= & \int_{0}^{1 / 2} \frac{(1-t)^{\alpha}-t^{\alpha}}{\left[t a^{p}+(1-t) b^{p}\right]^{1-\frac{1}{p}}} d t+\int_{1 / 2}^{1} \frac{t^{\alpha}-(1-t)^{\alpha}}{\left[t a^{p}+(1-t) b^{p}\right]^{1-\frac{1}{p}}} d t \\
= & \int_{0}^{1} \frac{t^{\alpha}-(1-t)^{\alpha}}{\left[t a^{p}+(1-t) b^{p}\right]^{1-\frac{1}{p}}} d t+2 \int_{0}^{1 / 2} \frac{(1-t)^{\alpha}-t^{\alpha}}{\left[t a^{p}+(1-t) b^{p}\right]^{1-\frac{1}{p}}} d t \\
\leq & \int_{0}^{1} \frac{t^{\alpha}}{\left[t a^{p}+(1-t) b^{p}\right]^{1-\frac{1}{p}}} d t-\int_{0}^{1} \frac{(1-t)^{\alpha}}{\left[t a^{p}+(1-t) b^{p}\right]^{1-\frac{1}{p}}} d t \\
& +2 \int_{0}^{1 / 2} \frac{(1-2 t)^{\alpha}}{\left[t a^{p}+(1-t) b^{p}\right]^{1-\frac{1}{p}}} d t
\end{aligned}
$$




$$
\begin{gathered}
(2.18)=M_{4}(\alpha, a, b)=\frac{b^{1-p}}{\alpha+1}\left[\begin{array}{c}
{ }_{2} F_{1}\left(1-\frac{1}{p}, \alpha+1 ; \alpha+2 ; 1-\frac{a^{p}}{b^{p}}\right)- \\
{ }_{2} F_{1}\left(1-\frac{1}{p}, 1 ; \alpha+2 ; 1-\frac{a^{p}}{b^{p}}\right) \\
+{ }_{2} F_{1}\left(1-\frac{1}{p}, 1 ; \alpha+2 ; \frac{1}{2}\left(1-\frac{a^{p}}{b^{p}}\right)\right)
\end{array}\right], \\
K_{5}=\int_{0}^{1} \frac{\left|(1-t)^{\alpha}-t^{\alpha}\right|}{\left[t a^{p}+(1-t) b^{p}\right]^{1-\frac{1}{p}}} t d t \\
\leq \int_{0}^{1} \frac{t^{\alpha+1}}{\left[t a^{p}+(1-t) b^{p}\right]^{1-\frac{1}{p}}} d t-\int_{0}^{1} \frac{(1-t)^{\alpha}}{\left[t a^{p}+(1-t) b^{p}\right]^{1-\frac{1}{p}}} t d t \\
\quad+2 \int_{0}^{1 / 2} \frac{(1-2 t)^{\alpha}}{\left[t a^{p}+(1-t) b^{p}\right]^{1-\frac{1}{p}}} t d t
\end{gathered}
$$$$
(2.19)=M_{5}(\alpha, a, b)=\frac{b^{1-p}}{\alpha+2}\left[\begin{array}{c}
{ }_{2} F_{1}\left(1-\frac{1}{p}, \alpha+2 ; \alpha+3 ; 1-\frac{a^{p}}{b^{p}}\right) \\
-\frac{1}{\alpha+1} F_{1}\left(1-\frac{1}{p}, 2 ; \alpha+3 ; 1-\frac{a^{p}}{b^{p}}\right) \\
+\frac{1}{2(\alpha+1)} F_{1}\left(1-\frac{1}{p}, 2 ; \alpha+3 ; \frac{1}{2}\left(1-\frac{a^{p}}{b^{p}}\right)\right)
\end{array}\right] \text {, }
$$

$$
\begin{aligned}
K_{6}= & \int_{0}^{1} \frac{\left|(1-t)^{\alpha}-t^{\alpha}\right|}{\left[t a^{p}+(1-t) b^{p}\right]^{1-\frac{1}{p}}}(1-t) d t \\
\leq & \int_{0}^{1} \frac{t^{\alpha}}{\left[t a^{p}+(1-t) b^{p}\right]^{1-\frac{1}{p}}}(1-t) d t-\int_{0}^{1} \frac{(1-t)^{\alpha+1}}{\left[t a^{p}+(1-t) b^{p}\right]^{1-\frac{1}{p}}} d t \\
& +2 \int_{0}^{1 / 2} \frac{(1-2 t)^{\alpha}}{\left[t a^{p}+(1-t) b^{p}\right]^{1-\frac{1}{p}}}(1-t) d t
\end{aligned}
$$

$(2.20)=M_{6}(\alpha, a, b)=\frac{b^{1-p}}{\alpha+2}\left[\begin{array}{c}\frac{1}{\alpha+1}_{2} F_{1}\left(1-\frac{1}{p}, \alpha+1 ; \alpha+3 ; 1-\frac{a^{p}}{b^{p}}\right) \\ -{ }_{2} F_{1}\left(1-\frac{1}{p}, 1 ; \alpha+3 ; 1-\frac{a^{p}}{b^{p}}\right) \\ +{ }_{2} F_{1}\left(1-\frac{1}{p}, 1 ; \alpha+2 ; \frac{1}{2}\left(1-\frac{a^{p}}{b^{p}}\right)\right) \\ -\frac{1}{2(\alpha+1)} F_{1}\left(1-\frac{1}{p}, 2 ; \alpha+3 ; \frac{1}{2}\left(1-\frac{a^{p}}{b^{p}}\right)\right)\end{array}\right]$.

Then by using the results from the equations (2.18)-(2.20), we have the desired inequality (2.15). This completes the proof. 
Theorem 2.4. Let $f: I \subset(0, \infty) \rightarrow \mathbb{R}$ be a differentiable function on $I^{\circ}, a, b \in I$ with $a<b, p>0$, and $f^{\prime} \in L[a, b]$. If $\left|f^{\prime}\right|^{q}$ is $p$-convex on $[a, b]$ for $q \geq 1$, then the following inequality for fractional integrals holds:

$(2.21 \mid) K_{f}(\alpha, a, b) \mid \leq \frac{b^{p}-a^{p}}{2 p} M_{7}^{1 / r}(\alpha, a, b)\left(\frac{1}{\alpha q+1}\right)^{1 / q}\left(\frac{\left|f^{\prime}(a)\right|^{q}+\left|f^{\prime}(b)\right|^{q}}{2}\right)^{1 / q}$

where

$$
M_{7}(\alpha, a, b)=\frac{b^{1-p}}{2} F_{2}\left(r-\frac{r}{p}, 1 ; 2 ; 1-\frac{a^{p}}{b^{p}}\right)
$$

and $1 / r+1 / q=1$.

Proof. From Lemma 1.2 and Lemma 2.1, by using the property of the modulus, the Hölder inequality and the p-convexity of $\left|f^{\prime}\right|^{q}$, we obtain,

$$
\begin{aligned}
& \left|K_{f}(\alpha, a, b)\right| \\
\leq & \frac{b^{p}-a^{p}}{2 p} \int_{0}^{1} \frac{\left|(1-t)^{\alpha}-t^{\alpha}\right|\left|f^{\prime}\left(\left[t a^{p}+(1-t) b^{p}\right]^{\frac{1}{p}}\right)\right|}{\left[t a^{p}+(1-t) b^{p}\right]^{1-\frac{1}{p}}} d t \\
\leq & \frac{b^{p}-a^{p}}{2 p}\left(\int_{0}^{1} \frac{1}{\left[t a^{p}+(1-t) b^{p}\right]^{r-\frac{r}{p}}} d t\right)^{1 / r} \\
& \left.\times \int_{0}^{1}\left|(1-t)^{\alpha}-t^{\alpha}\right|^{q}\left|f^{\prime}\left(\left[t a^{p}+(1-t) b^{p}\right]^{\frac{1}{p}}\right)\right|^{q} d t\right)^{1 / q} \\
\leq & \frac{b^{p}-a^{p}}{2 p}\left(\int_{0}^{1 / r} \frac{1}{\left[t a^{p}+(1-t) b^{p}\right]^{r-\frac{r}{p}}} d t\right)^{1 / q} \\
& \times\left(\int_{0}^{1}|1-2 t|^{\alpha q}\left[t\left|f^{\prime}(a)\right|^{q}+(1-t) t\left|f^{\prime}(b)\right|^{q}\right] d t\right)^{1 / q}\left(\frac{\left|f^{\prime}(a)\right|^{q}+\left|f^{\prime}(b)\right|^{q}}{2}\right)^{1 / q} \\
= & \frac{b^{p}-a^{p}}{2 p} M_{7}^{1 / r}(\alpha, a, b)\left(\frac{1}{\alpha q+1}\right)^{1 / 2}
\end{aligned}
$$

after calculations of integrals in the inequality (2.21) as follows,

$(2.24) M_{7}(\alpha, a, b)=\int_{0}^{1} \frac{1}{\left[t a^{p}+(1-t) b^{p}\right]^{r-\frac{r}{p}}} d t=\frac{b^{1-p}}{2} F_{2}\left(r-\frac{r}{p}, 1 ; 2 ; 1-\frac{a^{p}}{b^{p}}\right)$ 


$$
\begin{gathered}
\int_{0}^{1}|1-2 t|^{\alpha q} t d t=\int_{0}^{1 / 2}(1-2 t)^{\alpha q} t d t+\int_{1 / 2}^{1}(2 t-1)^{\alpha q} t d t=\frac{1}{2(\alpha q+1)} \\
\int_{0}^{1}|1-2 t|^{\alpha q}(1-t) d t=\frac{1}{2(\alpha q+1)} .
\end{gathered}
$$

Then by using the results from the equations (2.24)-(2.26) in the equation (2.23), then we have the desired result (2.21). This the completes the proof.

Theorem 2.5. Let $f: I \subset(0, \infty) \rightarrow \mathbb{R}$ be a differentiable function on $I^{\circ}, a, b \in I$ with $a<b, p>0$, and $f^{\prime} \in L[a, b]$. If $\left|f^{\prime}\right|^{q}$ is $p$-convex on $[a, b]$ for $q \geq 1$, then the following inequality for fractional integrals holds:

$$
\left|K_{f}(\alpha, a, b)\right| \leq \frac{b^{p}-a^{p}}{2 p}\left(M_{8}^{1 / r}(\alpha, a, b)+M_{9}^{1 / r}(\alpha, a, b)\right)\left(\frac{\left|f^{\prime}(a)\right|^{q}+\left|f^{\prime}(b)\right|^{q}}{2}\right)^{1 / q}
$$

where

$$
\begin{aligned}
& M_{8}(\alpha, a, b)=\frac{b^{(1-p) r}}{\alpha p+1_{2}} F_{1}\left(r-\frac{r}{p}, 1 ; \alpha r+2 ; 1-\frac{a^{p}}{b^{p}}\right) \\
& M_{9}(\alpha, a, b)=\frac{b^{(1-p) r}}{\alpha p+1_{2}} F_{1}\left(r-\frac{r}{p}, \alpha r+1 ; \alpha r+2 ; 1-\frac{a^{p}}{b^{p}}\right)
\end{aligned}
$$

and $1 / r+1 / q=1$.

Proof. From Lemma 2.1, by using the property of the modulus, the Hölder inequality and the p-convexity of $\left|f^{\prime}\right|^{q}$, then we obtain,

$$
\begin{aligned}
& \left|K_{f}(\alpha, a, b)\right| \\
\leq & \frac{b^{p}-a^{p}}{2 p} \int_{0}^{1} \frac{\left|(1-t)^{\alpha}-t^{\alpha}\right|\left|f^{\prime}\left(\left[t a^{p}+(1-t) b^{p}\right]{ }^{\frac{1}{p}}\right)\right|}{\left[t a^{p}+(1-t) b^{p}\right]^{1-\frac{1}{p}}} d t \\
\leq & \frac{b^{p}-a^{p}}{2 p}\left\{\begin{array}{l}
\left(\int_{0}^{1} \frac{(1-t)^{\alpha r}}{\left[t a^{p}+(1-t) b^{p}\right]^{r-\frac{r}{p}}} d t\right)^{1 / r}\left(\int_{0}^{1}\left|f^{\prime}\left(\left[t a^{p}+(1-t) b^{p}\right] \frac{1}{p}\right)\right|^{q} d t\right)^{\frac{1}{q}} \\
+\left(\int_{0}^{1} \frac{t^{\alpha r}}{\left[t a^{p}+(1-t) b^{p}\right]^{r-\frac{1}{p}}} d t\right)^{1 / r}\left(\int_{0}^{1}\left|f^{\prime}\left(\left[t a^{p}+(1-t) b^{p}\right]^{\frac{1}{p}}\right)\right|^{q} d t\right)^{\frac{1}{q}}
\end{array}\right\}
\end{aligned}
$$

$$
\leq \frac{b^{p}-a^{p}}{2 p}\left(M_{8}^{1 / r}(\alpha, a, b)+M_{9}^{1 / r}(\alpha, a, b)\right)\left(\int_{0}^{1} t\left|f^{\prime}(a)\right|^{q}+(1-t)\left|f^{\prime}(b)\right|^{q} d t\right)^{\frac{1}{q}}
$$

$$
=\frac{b^{p}-a^{p}}{2 p}\left(M_{8}^{1 / r}(\alpha, a, b)+M_{9}^{1 / r}(\alpha, a, b)\right)\left(\frac{\left|f^{\prime}(a)\right|^{q}+\left|f^{\prime}(b)\right|^{q}}{2}\right)^{1 / q},
$$


after calculations of integrals in the inequality (2.28) as follows,

$$
M_{8}(\alpha, a, b)=\int_{0}^{1} \frac{(1-t)^{\alpha r}}{\left[t a^{p}+(1-t) b^{p}\right]^{r-\frac{r}{p}}} d t=\frac{b^{(1-p) r}}{\alpha p+1}{ }_{2} F_{1}\left(r-\frac{r}{p}, 1 ; \alpha r+2 ; 1-\frac{a^{p}}{b^{p}}\right)
$$

$$
M_{9}(\alpha, a, b)=\int_{0}^{1} \frac{t^{\alpha r}}{\left[t a^{p}+(1-t) b^{p}\right]^{r-\frac{r}{p}}} d t=\frac{b^{(1-p) r}}{\alpha p+1}{ }_{2} F_{1}\left(r-\frac{r}{p}, \alpha r+1 ; \alpha r+2 ; 1-\frac{a^{p}}{b^{p}}\right) .
$$

Then by using the results from the equations (2.31)-(2.32) in the equation (2.29), then we have the desired inequality (2.28). This completes the proof.

\section{REFEREN CES}

1. M. Avci, H. Kavurmaci and M. E. Ozdemir, New inequalities of HermiteHadamard type via s-convex functions in the second sense with applications, Appl. Math. Comput., 217 (2011), 5171\{5176.

2. P. L. Butzer, A. A. Kilbas, and J. J. Trujillo. Compositions of hadamard-type fractional integration operators and the semigroup property, Journal of Mathematical Analysis and Applications, 269:387400, 2002.

3. Z. B. Fang and R. Shi, On the(p; h)-convex function and some integral inequalities, J. Inequal. Appl., 2014(45)(2014), 16 pages.

4. E. Guariglia, Fractional Derivate of Riemann-Zeta Function: in Fractional Dynamics, Cattani, Srivastava, Yang (Eds.), De Gruyter, pp 357-368,2015.

5. E. Guariglia and S. Silvestrov, Fractional-Wavelet Analysis of Positive definite Distributions and Wavelets on D'(C), in Engineering Mathematics II, Silvestrov, Rancic (Eds), Springer, pp 357-353, 2017.

6. I. Iscan, A new generalization of some integral inequalities for $(\alpha ; \mathrm{m})$-convex functions, Mathematical Sciences, 7(22) (2013),1-8.

7. I. Iscan, New estimates on generalization of some integral inequalities for s-convex functions and their applications, International Journal of Pure and Applied Mathematics,86(4) (2013), 727-746.

8. I. Iscan, Some new general integral inequalities for h-convex and h-concave functions, Adv. Pure Appl. Math. 5(1)(2014), 21-29.

9. I. Iscan, Ostrowski type inequalities for p-convex functions, New Trends in Mathematical Sciences, No. 3, 140-150 (2016).

10. I. Iscan, Hermite-Hadamard-Fejer type inequalities for convex functions via fractional integrals, Studia UniversitatisBabe s-Bolyai Mathematica, 60(3) (2015), $355-366$.

11. I. Iscan, Hermite-Hadamard type inequalities for harmonically convex functions, Hacettepe Journal of Mathematics and Statistics, 43(6) (2014), 935-942. 
12. I. Iscan, Hermite-Hadamard type inequalities for harmonically convex functions, Hacet. J. Math. Stat. 43 (6) (2014), 935-942.

13. U.N. Katugampola, New approach to a generalized fractional integral, Appl. Math. Comput. 218(3)(2011) 860-865.

14. U.N. Katugampola, New approach to generalized fractional derivatives, Bull. Math. Anal. Appl. 6(4)(2014) 1-15.

15. U.N. Katugampola, Mellin transforms of generalized fractional integrals and derivatives, Appl. Math. Comput. 257(2015)566-580.

16. A.A. Kilbas, H.M. Srivastava and J.J. Trujillo, Theory and applications of fractional differential equations, Elsevier,Amsterdam, 2006.

17. A. A. Kilbas, Hadamard-type fractional calculus, Journal of Korean Mathematical Society, 38(6):1191-1204, 2001.

18. U.S. Kirmaci, Inequalities for differentiable mappings and applications to special means of real numbers and to midpoint formula,Appl. Math. Comput. 147 (2004), 137-146.

19. V. Kiryakova. Generalized fractional calculus and applications, John Wiley and Sons Inc., New York, 1994.

20. K. S. Miller and B. Ross, An introduction to the fractional calculus and fractional diffrential equations, Wiley, New York, 1993.

21. M. A. Noor, K. I. Noor and S. Iftikhar, Nonconvex Functions and Integral Inequalities, Punjab University Journal of Mathematics, 47(2) (2015), 19-27.

22. M. A. Noor, K. I. Noor, M. V. Mihai, and M. U. Awan, Hermite-Hadamard inequalities for differentiable p-convex functions using hypergeometric functions, Researchgate doi: 10.13140/RG.2.1.2485.0648.

23. K. B. Oldham and J. Spanier, The fractional calculus, Academic Press, New York, 1974 .

24. A. Prudnikov, Y. Brychkov, O. Marichev, Integral and series. In: Elementary Functions,vol. 1. Nauka, Moscow; 1981.

25. S. G. Samko, A. A. Kilbas, and O. I. Marichev, Fractional Integrals and Derivatives: Theory and Applications, Gordon and Breach, Yverdon, 1993.

26. M.Z. Sarıkaya, E. Set, H. Yaldız, N.Basak, Hermite-Hadamard's inequalities for fractionalintegrals and related fractional inequalities, Mathematical and Computer Modelling, 57(2013)2403-2407.

27. G. K. Srinivasan, The gamma function: An Eclectic Tour, Amer. Math. Monthly 114, 297-315 (2007).

28. K.S. Zhang and J.P. Wan, p-convex functions and their properties, Pure Appl. Math. 23(1) (2007), 130-133..

29. J. Wang, C. Zhu, Y. Zhou. New generalized Hermite-Hadamard type inequalities and applications to special means. J. Inequal. Appl. 2013;2013(325):1-15 
Tekin Toplu

Faculty of Arts and Sciences

Department of Mathematics

P.O. Box 28100

Giresun, Turkey

tekintoplu@gmail.com

Erhan Set

Faculty of Arts and Sciences

Department of Mathematics

P.O. Box 52200

Ordu, Turkey

erhanset@yahoo.com

İmdat İşcan

Faculty of Arts and Sciences

Department of Mathematics

P.O. Box 28100

Giresun, Turkey

imdati@yahoo.com

Selahattin Maden

Faculty of Arts and Sciences

Department of Mathematics

P.O. Box 52200

Ordu, Turkey

maden55@mynet.com 